Int. J. Morphol.,

32(1):147-150, 2014.

\title{
Descripción Anatómica y Funcional del Húmero del Tití Gris (Saguinus leucopus)
}

\author{
Anatomical and Funcional Description of Humerus in the White-Footed Tamarin (Saguinus leucopus)
}

\author{
Jorge Eduardo Duque Parra,*** \& Juan Fernando Vélez García****
}

DUQUE PARRA, J. E. \& VÉLEZ GARCÍA, J. F. Descripción anatómica y funcional del húmero del tití gris (Saguinus leucopus). Int. J. Morphol., 32(1):147-150, 2014.

RESUMEN: El Saguinus leucopus es un primate neotropical, endémico y monotípico de Colombia, con escasos estudios sobre su anatomía, cuya morfología humeral permanece desconocida siendo un elemento fundamental para la locomoción cuadrúpeda de este animal arbóreo. El objectivo del presente estudio fue describir la anatomía macroscópica y la función de los relieves óseos del húmero del titi gris Saguinus leucopus. Se tomaron como base del estudio ocho especímenes fijados con formalina al 10\% y de ellos se extrajeron sus húmeros, describiendo las principales funciones de los relieves óseos, como fijaciones musculares y canalizaciones neurovasculares. Los variados relieves anatómicos del húmero del titi gris presentan una disposición morfológica coincidente con sus hábitos de locomoción cuadrúpeda arbórea.

PALABRAS CLAVE: Anatomía; Osteología; Húmero; Primate.

\section{INTRODUCTION}

El titi gris (Saguinus leucopus) es un primate neotropical, endémico y monotípico de Colombia (Defler, 2010), que se distribuye geográficamente entre la parte baja del río Cauca y el valle medio del río Magdalena, correspondiente a los departamentos de Antioquia, Caldas, sur de Bolívar y norte de Tolima (Hernández \& Defler, 1983; Leal et al., 2010). Su postura y locomoción son cuadrúpedas, se moviliza principalmente con saltos, y presenta la capacidad de suspenderse y adherirse a las ramas y troncos de los árboles (Defler). Los estudios morfológicos del húmero siguen siendo realizados y en ellos se toman como referentes los diversos relieves óseos como tuberosidades, crestas, cabeza (Somesh et al., 2011), tróclea (Thewissen et al., 2001) entre otros, que permiten a los anatomistas (Somesh et al.) y otros profesionales, tener una mejor comprensión de este hueso del miembro torácico, ya que las diversas y complejas formas deben ser buscadas para entender sus relaciones con la función (Ross, 2005) en primates (Cossins, 2013). En ello un acercamiento a la locomoción incluyendo algunos primates que son cuadrúpedos (Yamanaka et al., 2005), y no se escapa de ello, el estudio de la morfología animal, como es el caso del tití gris (Saguinus leucopus).
Son escasos los estudios que se encuentran sobre la anatomía del titi gris (Stevenson et al., 2010), por lo que lograr un conocimiento de sus características morfológicas, contribuirá al significado funcional (Aversi-Ferreira et al., 2005a), como es el caso del esqueleto apendicular utilizado para la locomoción (Alexander, 1994), en el que los principales elementos para estos roles son los huesos longos (Marieb \& Mallat, 1992) como el húmero (Erickson et al., 2002). Estos conocimientos, además sirven para poder explicar las diversas adaptaciones (Aversi-Ferreira et al., 2007) de los primates a su entorno, como en el caso de los primates del nuevo mundo, que son una diversa radiación de primates no humanos que ocupan amplio rango de hábitats en América, desde México hasta Argentina (Youlatos \& Meldrum, 2012) incluida Colombia. Los primates del nuevo mundo dependen principalmente de frutas y para ello requieren de trepar y en ello, tener patrones de locomoción (Strier, 1992). En la exploración de sus diversos nichos, requieren conductas de posición que los capacite para la manipulación, ingestión y escape de potenciales depredadores (Garber, 2007). Al fin y al cabo el comportamiento es siempre el resultado final de la integración complejísima de contracciones musculares (Tinbergen, 1969).

\footnotetext{
* Programa de Medicina, Departamento de Ciencias Básicas, Universidad de Caldas, Manizales, Colombia.

** Departamento de Biología, Universidad Autónoma de Manizales, Manizales, Colombia.

${ }^{* * * *}$ Programa de Medicina Veterinaria y Zootecnia, Departamento de Salud Animal, Universidad de Caldas en convenio con CORPOCALDAS, Manizales, Colombia.
} 
Por lo tanto, el estudio de la locomoción animal es fundamental para el entendimiento de su adaptación (Youlatos \& Meldrum) y éste deriva del aparato locomotor en el que se incluye el soporte óseo del brazo. La caracterización anatómica del húmero aporta información relevante, ya que una de las visiones centrales de los vertebrados son las consecuencias funcionales atendiendo a la diversidad morfológica del esqueleto (Ross). Por lo tanto, el conocimiento de las condiciones morfológicas, especialmente de animales permitirán posteriores estudios funcionales (Marques et al., 2006). Hay descripciones anatómicas de la musculatura de primates neotropicales (Aversi-Ferreira et al., 2005b, 2007), quienes describen origen e inserción de los músculos del miembro torácico en otras especies como Cebus libidinosus, y Cebus apella (Aversi-Ferreira et al., 2005a, 2006) y Cebus albifrons (Cribillero et al., 2009). La musculatura del miembro torácico comprende los músculos del cíngulo escapular -los cuales se encuentran entre el tronco y el miembro torácico- y los músculos intrínsecos, los cuales poseen su origen e inserción en las regiones propias del miembro torácico (Dyce et al., 2007) como lo es en el húmero.

\section{MATERIAL Y MÉTODO}

Se utilizaron ocho especímenes de Tití gris (Saguinus leucopus), entre ellos cuatro hembras y cuatro machos con pesos entre 300 y 460 gramos que murieron por causas naturales entre el año 2012 y primer trimestre del 2013 en los centros de atención y valoración de fauna silvestre de CORPOCALDAS. Se fijaron mediante infiltraciones subcutáneas, intramusculares y en cavidades con una solución de formalina al $10 \%$, aceite mineral al $5 \%$ y ácido fénico al $1 \%$.
Posteriormente se sumergieron en la misma solución por un periodo mínimo de 48 horas. Se disecaron desde el plano superficial al profundo y se llevaron registros fotográficos del procedimiento en el que se enfatizó la anatomía y función del húmero, cuyos hallazgos anatómicos se describieron según la terminología de la Nómina Anatómica Veterinaria del 2012 (ICVGAN, 2012) y la Terminología Anatómica Internacional de 1998 (FICAT, 1998).

\section{RESULTADOS}

El húmero del S. leucopus presenta relieves para fijaciones musculares, ligamentosas, cursos neurovasculares y zonas cartilaginosas para diartrosis con otras partes del miembro torácico. En la epífisis proximal la cabeza dirigida hacia caudal, articula con la escápula. Dos tubérculos: uno medial y otro lateral para fijaciones del músculo subescapular en el medial y en el lateral los músculos supraespinoso e infraespinoso, que se inserta en una depresión del tubérculo. Un canal intertubercular proyectado más del tercio proximal humeral para el tendón de la cabeza longa del bíceps braquial (Figura 1). En las crestas de los tubérculos menor y mayor se insertan respectivamente los músculos terete mayor y menor. En el tercio superior de la diáfisis una uve como cresta para el músculo deltoides.

El nervio radial rodea la diáfisis, mas no hay un surco para dicho nervio. La epífisis distal aplanada cráneocaudalmente presenta la tróclea que articula con la ulna, lateralmente un capítulo para el radio. Lateral al capítulo un epicóndilo con una pronunciada cresta supracondílea lateral cerca de un tercio de la diáfisis (Fig. 1) y en la que se fijan

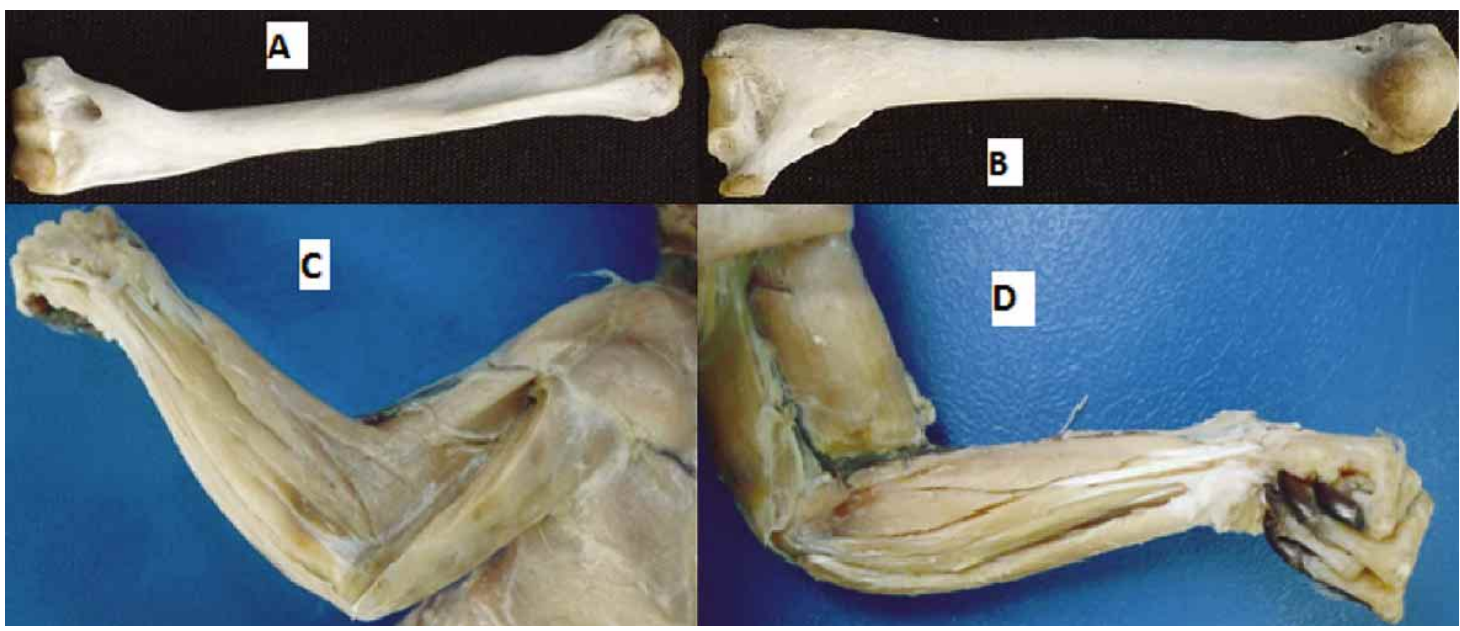

Fig. 1. En A: Húmero izquierdo de S. leucopus con marcado epicóndilo medial para fijaciones musculares. Conducto supracondíleo para el nervio mediano. En B: Musculatura lateral antebraquial de S. leucopus. Se ha retirado parte de la cabeza lateral del tríceps braquial para notar fijaciones humerales. En C: Vista caudal del húmero izquierdo del $S$. leucopus. En D: Vista medial de la musculatura del epicóndilo medial del antebrazo de un S. leucopus. 
músculos como el braquioradial, el extensor radial longo entre otros (Fig. 1). Medial a la tróclea el epicóndilo medial muy pronunciado para músculos flexores antebraquiales (Fig. 1). Proximal y craneal a la tróclea, las fosas coronoidea y radial, esta última más amplia, que reciben el proceso coronoideo ulnar y la cabeza radial respectivamente (Fig. 1). Entre la tróclea y el epicóndilo medial un surco para curso del nervio ulnar. En la parte caudo-proximal troclear la fosa olecraneana que recibe el olecranon (Fig. 1). Por la región cráneo-medial de la epífisis distal un conducto supracondíleo, a través del cual este se canaliza el nervio mediano y la arteria ulnar (Fig. 1).

\section{DISCUSIÓN}

Descripciones del húmero de especies de locomoción similar a S. leucopus han sido documentadas y coinciden en muchas características a lo que encontramos en esta especie. Así, se describe el húmero del Callimico goeldii, que coincide en todos los relieves anatómicos, excepto que en la parte craneal de la epífisis distal hay una fosa supratroclear (Osman-Hill, 1959), pues el S. leucopus presenta la fosa radial y la coronoidea. El húmero del S. leucopus concuerda en que los primates hominoides tienen una articulación distal humeral perpendicular al cuerpo, más la cabeza del húmero se dirige hacia caudal y no hacia dorsomedial como en el humano (Ankel-Simons, 2007). En el húmero de varias especies de primates la cabeza siempre se dirige hacia caudo-medial, pero en Lemuroidea la cabeza se dirige hacia caudal (Mivart, 1867) igual que sucede en S leucopus. En $S$. oedipus especie del mismo género de $S$. leucopus con un patrón de locomoción similar como cuadrúpedo arbóreo (Defler \& Bueno, 2010), se han descrito los relieves anatómicos del húmero y se ha realizado un análisis funcional comparado con otros primates y se pudo determinar que el húmero del $S$. oedipus presenta un gran epicóndilo medial, como origen de músculos flexores de carpo y algunos de los dedos, ya que la mano se puede encontrar en diferentes grados de pronación y supinación durante su locomoción, pero este epicóndilo es menos extenso medialmente comparado con primates de locomoción suspensoria como Ateles sp. (Ackerman, 2003).
En la parte disto-medial humeral de primates del género Saguinus, se encuentra un foramen entepicondíleo por el que se canalizan el nervio mediano y la arteria braquial (AnkelSimons), pero por su morfología en S. leucopus lo describimos como conducto. En Cebus libidinosus (Aversi-Ferreira et al., 2007) al igual que en S. leucopus se encontró que la arteria que pasa a través de este conducto es la ulnar, pues ésta y la radial, ya están separadas en el brazo. En los mamíferos domésticos reportados en la nómina anatómica veterinaria (ICVGAN), solo el gato doméstico presenta foramen supracondíleo por donde pasan el nervio mediano y la arteria braquial (Dyce et al.) y por la descripción del húmero de éste (De luliis \& Pulera, 2007) hay similitud con el de S. leucopus con la cabeza y con todos los relieves de la epífisis distal, corroborando sus adaptaciones a la locomoción cuadrúpeda.

\section{CONCLUSIÓN}

Los múltiples relieves óseos del húmero del Tití gris (Saguinus leucopus) entre los que se notan hacia la epífisis proximal la cabeza humeral, los tubérculos medial y lateral, el surco intertubercular, son equiparables en parte a los del ser humano por comparación siempre y cuando se tenga en cuenta que su cabeza se dirige hacia caudal. Hacia la epífisis distal la tróclea, el epicóndilo medial, la cresta supracondílea medial, el epicóndilo lateral, la cresta supracondílea lateral, la fosa olecraneana, la fosa radial, la fosa coronoidea, el conducto supracondíleo, el surco para el nervio ulnar son equiparables más al gato doméstico que al humano y coinciden con elementos vasculares, nerviosos y musculares entre otros. Estos relieves óseos le permiten desarrollar sus funciones de movimiento en concordancia con el ambiente en el que se desenvuelven para la fijación a los troncos y ramas de los árboles en posición cuadrúpeda. Por último, debido a esta forma de locomoción las descripciones anatómicas que se realicen en estos primates deberían ser de acuerdo a los términos direccionales definidos para cuadrúpedos en la nómina anatómica veterinaria, y para realización de diagnósticos radiográficos y cirugías correctivas del húmero en esta especie se podrían utilizar las mismas técnicas que para el gato doméstico.

DUQUE PARRA, J. E. \& VÉLEZ GARCÍA, J. F. Anatomical and functional description of humerus in the White-footed tamarin (Saguinus leucopus). Int. J. Morphol., 32(1):7147-150, 2014.

SUMMARY: The Saguinus leucopus is a neotropical primate, endemic and monotypic of Colombia, with few studies reporting its anatomy. The humeral morphology for this species remains unknown and is a key element for the quadruped locomotion of this arboreal animal. The objective of the present study is to describe the gross anatomy and function of the humerus bone in the White-footed tamarin (Saguinus leucopus). The study was based on eight specimens, fixed in $10 \%$ formalin and from which we extracted the humeri, describing the main features of the bony prominences, such as muscle attachments and neurovascular pipes. The various anatomical contours of the humerus of Saguinus leucopus exhibit morphological disposition, matching their arboreal quadrupedal and locomotion habits. 


\section{REFERENCIAS BIBLIOGRÁFICAS}

Ackerman, R. A Comparative primate anatomy dissection manual. University of Capetown, 2003. Available in: http://web.uct.ac.za/ depts/age/people/dissect.pdf

Alexander, R. Bones: the unity ofform and function. London, Weindenfeld and Nicholson, 1994.

Ankel-Simons, F. Primate anatomy: An Introduction. Amsterdam, Elsevier, 2007.

Aversi-Ferreira, T. A.; Pereira-de-Paula, J.; Prado, Y. C. L.; Lima-e-Silva, M. S. \& Mata, J. R. Anatomy of the shoulder and arm muscles of Cebus libidinosus. Braz. J. Morphol. Sci., 24(2):63-74, 2007.

Aversi-Ferreira, T. A.; Roqueline, A. G.; Aversi-Ferreira, R. A.; Silva, Z.; Gouvêa-E-Silva, L. F. \& Penha-Silva, N. Estudo anatômico de músculos profundos do antebraço de Cebus apella (Linnaeus, 1766). Acta Sci. Biol. Sci., 27(3):297-301, 2005 a.

Aversi-Ferreira, T.A.; Lima-e-Silva, M. S.; Pereira-De-Paula, J.; GouvêaE-Silva, L. F. \& Penha-Silva, N. Anatomia comparativa dos nervos do braço de Cebus apella. Descrição do músculo dorsoepitroclear. Acta Sci. Biol. Sci., 27(3):291-6, 2005 b.

Aversi-Ferreira, T. A.; Vieira, L. G.; Pires, R. M.; Silva, Z. \& PenhaSilva, N. Estudo anatômico dos músculos flexores superficiais do antebraço no macaco Cebus apella. Biosci. J., 22(1):139-44, 2006.

Cossins, D. Monster Hunting 2.0. The Scientist. 2:20-1, 2013.

Cribillero, C. N.; Sato, S. A. \& Navarrete, Z. M. Anatomía macroscópica de la musculatura del miembro anterior del mono machín blanco (Cebus albifrons). Rev. Investig. Vet. Perú, 20(2):143-53, 2009.

De luliis, G. \& Pulera, D. The Dissection of Vertebrates: A Laboratory Manual. Amsterdam, Elsevier/Academic Press, 2007.

Defler, T. R. Historia Natural de Primates Neotropicales. Bogotá, Universidad Nacional de Colombia, 2010.

Defler, T. R. \& Bueno, M. L. Prioridades en investigación y conservación de primates colombianos. Primatología en Bogotá, Avances al Principio del Milenio, 2010.

Dyce, K. M.; Sack, W. O. \& Wensing, C. J. G. Anatomía veterinaria. México D.F., El Manual Moderno, 2007.

Erickson, G. M.; Catanese, J. 3rd. \& Keaveny, T. M. Evolution of the biomechanical material properties of the femur. Anat. Rec., 268(2):115-24, 2002.

Federative International Committee on Anatomical Terminology (FICAT). Terminologia Anatomica. International Anatomical Terminology. New York, Thieme, 1998.

Garber, P. A. Primate locomotor behavior and ecology. In: Campbell, C.; Fuentes, A.; MacKinnon, K. C.; Panger, M. \& Bearder, S. (Eds.). Primates in perspective. Oxford, Oxford University Press, 2007. pp.543-60.
Hernández, J. \& Defler, T. Algunos aspectos de la conservación de primates no humanos en Colombia. En: Saavedra, C.; Mittermeier, R. \& Santos, I. B. (Eds.). La primatología en Latinoamérica. Washington D.C., World Wildlife Foundation, 1983. pp.67-97.

International Committee on Veterinary Gross Anatomical Nomenclature (ICVGAN). Nomina Anatomica Veterinaria. Hannover, World Association of Veterinary Anatomists, 1992.

Leal, A.; Granados, J. L.; Zerda, E. \& Brieva, C. Liberación y seguimiento de dos grupos de tití gris (Saguinus leucopus) en el departamento del Tolima. Rev. Med. Vet. Zoot., 57(2):132-43, 2010.

Marieb, E. N. \& Mallatt, J. Human Anatomy. Redwood City, Benjamin/ Cummings Publishing Co., 1992.

Marques, M. A.; Vasconcellos, H. A. \& Azevedo, N. L. The union between gracilis and sartorius muscles in leontopithecus: Morphofunctional analysis. Int. J. Morphol., 24(2):215-20, 2006.

Mivart, G. On the appendicular skeleton of the primates. Phil. Trans. $R$. Soc. B., 157:299-429, 1867.

Osman-Hill, W. C The Anatomy of Callimicogoeldii (Thomas). Trans. Am. Philos. Soc., 49(5):1-116, 1959.

Ross, C. F. Finite elements analysis in vertebrate biomechanics. Anat. Rec. A Discov. Mol. Cell Evol. Biol., 283(2):253-8, 2005.

Somesh, M. S.; Prabhu, L. V.; Shilpa, K.; Pai, M. M.; Krishnamurthy, A. \& Murlimanju, B. V. Morphometric study of the humerus segments in India Population. Int. J. Morphol., 29(4):1174-80, 2011.

Stevenson, P. R.; Guzmán, D. C. \& Defler, T. R. Conservation of Colombian primates: an analysis of published research. Trop. Conserv. Sci. 3(1):45-62, 2010.

Strier, K. B. Atelinae adaptations: behavioral strategies and ecological constraints. Am. J. Phys. Anthropol., 88(4):515-24, 1992.

Thewissen, J. G.; Williams, E. M.; Roe, L. J. \& Hussain, S. T. Skeletons of terrestrial cetaceans and the relationship of whales to artyodactyls. Nature, 413(6853):277-81, 2001.

Tinbergen, N. El estudio del instinto. México D.F., Siglo XXI Editores, 1969.

Yamanaka, A.; Gunji, H. \& Ishida, H. Curvature, length, and crosssectional geometry of the femur and humerus in antrophoid primates. Am. J. Phys Antrophol., 127(1):46-57, 2005.

Youlatos, D. \& Meldrum, J. Locomotor Diversification in New World Monkeys: Running, Climbing, or Clawing Along Evolutionary Branches. Anat. Rec., 294(12):1991-2012, 2011.

Dirección para Correspondencia:

Jorge Eduardo Duque Parra

Programa de Medicina

Universidad de Caldas

Manizales - COLOMBIA

Recibido : 03-04-2013

Aceptado: $17-12-2013$

Email: jorge.duque_p@ucaldas.edu.co 